\title{
Familial occurrence of intracranial arterial occlusive disease (Moyamoya) in neurofibromatosis
}

\author{
Robert P. Erickson, 1,2 James Woolliscroft3 and Richard J. Allen 2,4 \\ Departments of 1 Human Genetics, 2 Pediatrics, 3 Internal Medicine, and 4 Neurology, \\ University of Michigan School of Medicine, Ann Arbor, Michigan, U.S.A.
}

Two out of six siblings with neurofibromatosis (in a sibship of eight) had clinical and roentgenographic evidence of Moyamoya-type, intracranial arterial occlusive disease. This rare vascular complication of neurofibromatosis has not previously occurred among primary relatives. Several possible etiologies for such an association are discussed.

Received 5 March, accepted for publication 22 April 1980

Key words: Intracranial arterial occlusive disease; Moyamoya disease; neurofibromatosis.

Moyamoya is a descriptive term for the cerebral angiographic finding of large arterial vessel occlusions and a telangiectatic vascular network involving the collateral circulation, usually in the area of the basal ganglia. The term was first used by Suzuki \& Takaku (1969) in describing the angiographic findings in a group of young Japanese patients with acute hemiparesis - the term Moyamoya indicates haziness, like that of a thin cloud. Although originally thought to be a specific, progressive entity, it has now been found in a variety of situations, including post-trauma, post-meningitis, with tuberculosis, with periarteritis nodosa, and with sickle-cell anemia (Hilal et al. 1971a, Seeler et al. 1978). It has also been found in the neurocutaneous disorders (Hilal et al. 1971b), but it is a rare occurrence in neurofibromatosis. Tomsick et al. (1976) reviewed 12 cases and added two of their own; Holt (1978), in his thorough review of radiologic manifestations of neurofibromatosis, added two more cases; and single or double case reports continue to appear (Götze \& Kühne 1976, Lamas et al. 1978, Levisohn et al. 1978). None of these reports of Moyamoya in neurofibromatosis have included cases within one family. We report siblings with both neurofibromatosis and intracranial arterial occlusive disease.

\section{Case Reports}

Family History (Pedigree in Fig. 1)

Some members in three generations of the $P$ family showed classic signs of neurofibromatosis, including multiple pigmented spots and skin "tumors". The stigmata were well recognized by the family but they were unable to trace the disease to earlier ancestors, I-2 being the first known affected. Cases III-4 and III-7 are reported in detail because of their manifestations of multiple intracranial arterial ocolusive (Moyamoya) disease, while their father's neurological 


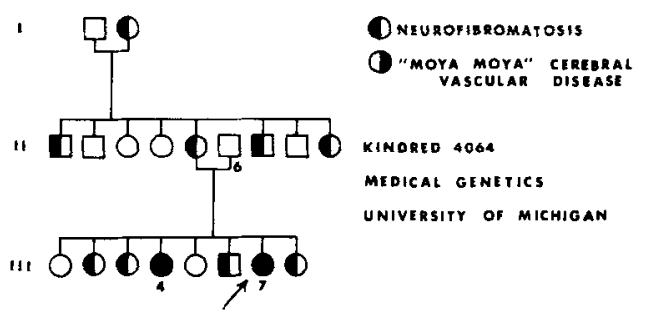

Fig. 1. Pedigree of $P$ family. Roman numerals indicate generation number; arabic numerals indicate the individual discussed.

history (II-6) is briefly recorded because of its possible relevance.

Case III-4 (age 26 years). This female patient was well, although she had cutaneous manifestations of neurofibromatosis, until the age of 5 years when she had a sudden onset of right-sided weakness and aphasia. The episode lasted about an hour and left no residua. About 6 months later she had an acute episode of right-sided paralysis, which also subsided rapidly. A third acute episode, several months later, involved partial left hemiparesis and again cleared. Cerebral arteriography showed left cerebral atrophy with a widespread vascular anomaly consisting of multiple, small AV communications with small capillary filling providing a mulberry appearance. There were also mottling and irregularity of the left middle cerebral artery.

The patient required special education but achieved a special high school diploma. Her present height is $152.5 \mathrm{~cm}$ ( $5 \%$ ile). She has numerous cutaneous soft, lumpy swellings, numerous café-au-lait spots, and considerable freckling, which combine to create an appearance of increased age. There is slight skull asymmetry, with the left frontal and maxillary bones being larger than those on the right. Ophthalmological examination revealed 10-20 Lisch spots on the irides and a similar number of small choroidal cafẹ́-au-lait spots. Neuro- logical examination revealed extensive weakness distally on the right, most marked in the upper extremity, despite being righthanded. The reflexes in the right extremities were slightly more active than on the left; there was no spread of reflexes from clavicular percussion but there was some finger flexion with right biceps and triceps percussion. Babinski testing, but not Chaddock, showed an extensor toe sign on the right, while the reverse was true on the left.

At age 26, computerized-tomography brain scan showed loss of brain substance in the left cerebral hemisphere with moderate dilation of the left lateral ventricle and a shift of midline structures toward the left (Fig. 2). There was a pathologic radiolucency involving the left cerebral cortex region, extending from the temporal lobe anteroinferiorly, almost to the vertex of the skull, along sulcal margins and not involving white matter. The electroencephalogram was mildly abnormal and paroxysmal with a predominantly left-sided gradient.

Case 1II-7 (age 12 years). This female patient was well, although she had cutaneous

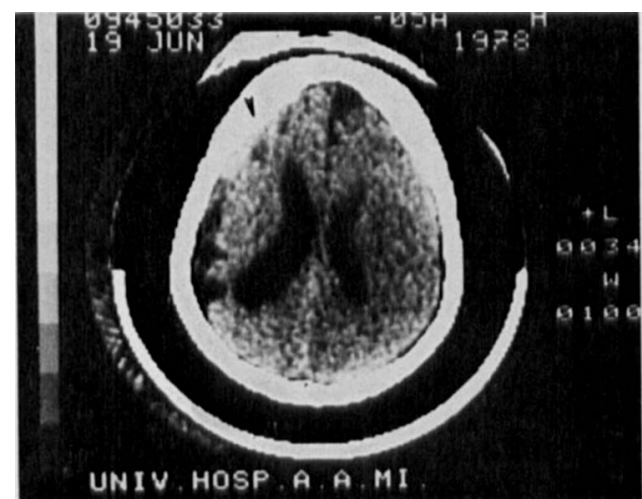

Fig. 2. Contrast computerized brain tomography of patient III-4, performed at age 26 years, showing loss of brain substance in the left cerebral hemisphere (arrow) with moderate dilation of the left lateral ventricle and a shift of midline structures towards the lẹtt. 


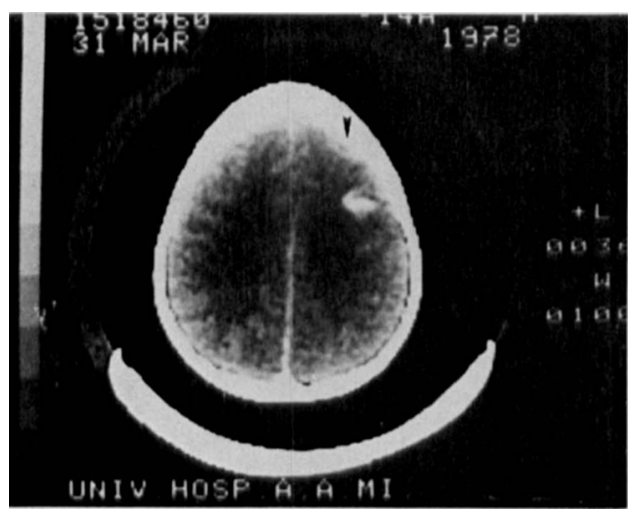

Flg. 3. Contrast computerized brain tomography of patient III-7 performed at age 11 years showing an enhancing lesion in the posterior right frontal convexity (arrow).

manifestations of neurofibromatosis, until age 11 when she noted the acute onset of tonic-clonic spasms of the left hand. The spasms lasted for 2 hours, and over the next 3 days moderate spastic paresis of the left hand developed. A moderate frontal headache during movement of the head developed at the same time. There was a questionable history of several short episodes of trouble straightening the left fingers during the 3 preceding years. Physical examination at this time showed height 131 $\mathrm{cm} \quad(<3 \%$ ile), and weight $29.5 \mathrm{~kg}$ $(<3 \%$ ile). There were numerous large, brown, café-au-lait spots over the thorax and extremities without any other lesions being evident, but there were no palpable neurofibromas. Examination of the eyes showed a single Lisch spot on the inferior right iris and a single nevus on the superior right retina. Other positive physical findings were limited to the neurological exam. Biceps, wrist extensor, and wrist flexor strength were moderately decreased on the left, while hand grip and interossei muscles were markedly weak on the left. The left hand was held with the metacarpophalangeal joints in hyperextension with the pa- tient unable to extend the proximal interphalangeal joint. Deep tendon reflexes were symmetrical and the Babinski sign was absent.

As part of her work-up, computerized brain tomography was performed with and without enhancement. A lesion compatible with an arteriovenous malformation was noted in the posterior right frontal convexity region (Fig. 3). The electroencephalogram revealed a focal disturbance in the right parasaggital area, which affected right central, parietal, and occipital areas. Because of these findings, a right common carotid, right vertebral and left internal carotid arteriogram was also performed, during general anesthesia. The supraclinoid segment of the right internal carotid artery was severely narrowed. Antegrade flow to the right middle and anterior cerebral arteries was poor and, in at least two areas,

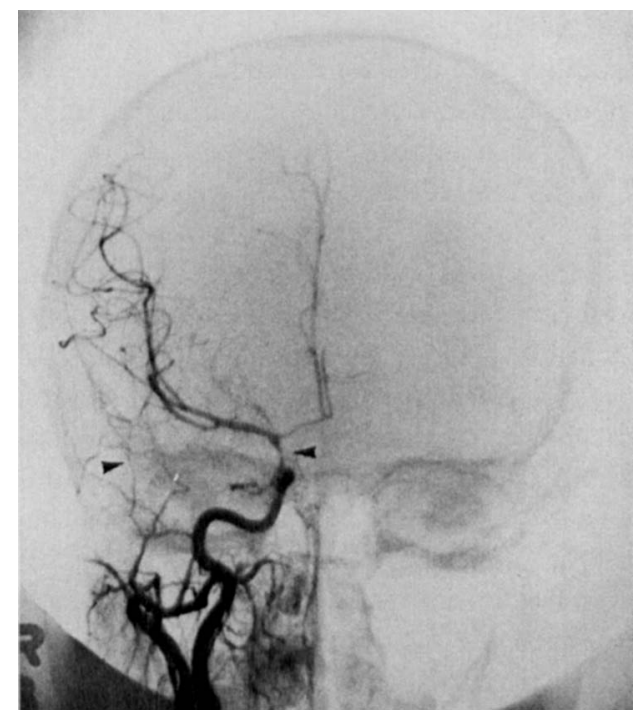

Fig. 4. Right common carotid arteriography of patient III-7 showing marked narrowing of the supraclinoid segment of the right internal carotid artery (large arrow) as well as right middle meningeal colfaterals to the right middle cerebral artery (small arrow). 


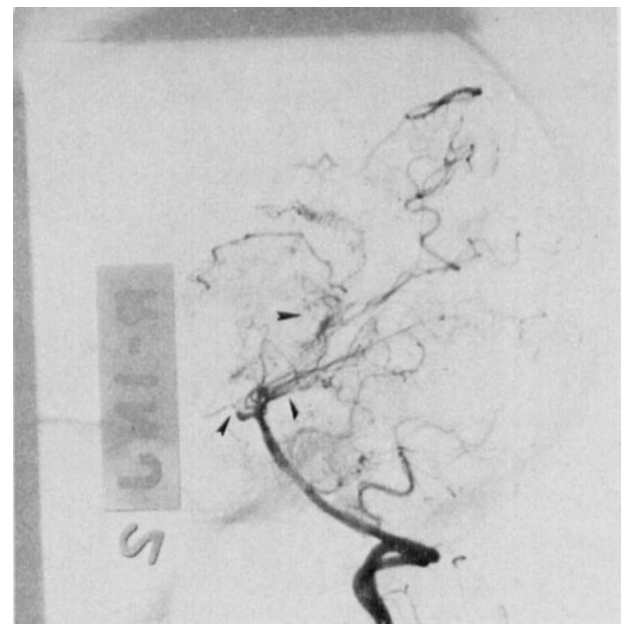

Fig. 5. Right vertebral-basilar arteriogram (patient III-7) showing poor filling of the right posterior cerebral artery (left lower arrow), enlargement of the right thalamoperforating and right posterior medial and lateral choroidal arteries (right lower arrow), and marked collateral extensions of the left posterior cerebral artery into the pericallosal and callosomarginal vessels (upper arrow).

branches of the right middle meningeal artery were seen to provide transmeningeal collateral flow to cerebral vessels of the right middle cerebral artery peripheral territory (Fig. 4). The right vertebral-basilar arteriogram showed poor filling of the right posterior cerebral artery, presumably due to occlusion (Fig. 5). There was marked enlargement of right thalamoperforating arteries and right posterior medial and lateral choroidal arteries (Fig. 5), presumably to provide collateral flow to the basal ganglia and choroid plexus. Marked collateral extensions of the left posterior cerebral artery into the pericallosal and callosomarginal vessels was also noted (Fig. 5). The left internal carotid angiography showed severe narrowing of the left anterior and middle cerebral arteries. In numerous areas there were small collections of tangledappearing tiny vessels which represent at- tempted transcerebral collateralization. The resulting pattern was that of multiple progressive intracranial occlusions.

The patient was started on $200 \mathrm{mg} /$ day of diphenylhydantoin and no recurrence of seizures was noted in the ensuing 15 months. She continued to do well in school, receiving B's and C's. There was some continued dysfunction of the left 4 th and 5th digits when attempting complex tasks such as typing.

Case II-6 (age 51 years). The father of the sisters with Moyamoya cerebral vascular abnormalities and neurofibromatosis had an 11-year history of diffuse neurological complaints. These consisted of intermittent pain and numbness in his hands, neck, and shoulders, with one episode of paralysis involving the entire left side. Neurological exam at another medical center at age 43 showed fine intention tremors of both hands with right deltoid, biceps, and triceps weakness. Tendon reflexes were decreased in the upper extremities and increased in the lower extremities, with bilateral flexor plantar responses. Complete blood count, various blood chemistries, and spinal fluid protein were within normal limits; the CSF gold curve was 0122100000 while VDRL was negative. Cervical myelography was normal. A presumptive diagnosis of multiple sclerosis was made and he was treated with high dose ACTH for a week (tapered) with some improvement. A year later, severe low back and right hip pain resulted in hospitalization. Physical findings were compatible with lumbar disc disease and treatment with muscle relaxants and traction resulted in marked improvement. An electroencephalogram performed at that time was within normal limits. The patient was gradually able to return to normal activities.

Physical examination at age 51 showed no evidence of sensory defect and little evidence of muscular weakness, although the right plantar reflex was extensor. 


\section{Discussion}

A great variety of vascular lesions have been found in neurofibromatosis. One of these causes hypertension in children, due to proximal renal artery stenosis, and is frequently associated with coarctation of the abdominal aorta (Holt 1978). Pulmonic stenosis, valvular and non-valvular, thoracic coarctation (Rowen et al. 1975), and fibromuscular stenosis of the right ventricular cavity (Rosenquist et al. 1970) have also been reported. It is a moot point whether secondary degenerative fibrosis or mesodermal dysplasia of vascular smooth muscle is responsible (Holt 1978).

Moyamoya disease was originally considered to be a distinctive, progressive entity, but it has since been found in a variety of situations and it has frequently been static (see Introduction). The clinical presentation is often that of cerebrovascular attacks, as in this family. Moyamoya has occasionally occurred in siblings; in one case report, 3 of 14 siblings were affected ( $\mathrm{\phi} \phi$ gaard \& Jørgensen 1975). However, even these familial cases have not been reported to have features typical of neurofibromatosis. On the other hand, intracranial arterial occlusive disease has been a rare complication of neurofibromatosis and multiple cases have not been previously reported in one family. It seems unlikely that the cooccurrence is due to chance - several possible etiological factors can be mentioned: (1) Moyamoya occurs more frequently in women and appears most commonly during childhood or adolescence. Since our patients were female and had their occurrence at the time of peak incidence, it seems possible that whatever the predisposing factors are in the general population, they interacted with the dominant neurofibromatosis gene to cause this rare complication. Thus, the familial occurrence would be for the same (unknown) reasons as the general familial occurrence of Moyamoya; (2) The father's non-definitively diagnosed neurological problems (which might be vascular or demyelinating) may have been genetic, and interaction of two dominant genetic disorders may have occurred; (3) There is genetic heterogeneity in neurofibromatosis, with one allele having a higher incidence of Moyamoya-type, intracranial vascular occlusive disease.

Pathological examination of intracranial arteries in a case of Moyamoya disease with neurofibromatosis (Lamas et al. 1978) has shown fibrous intimal thickening, reduplication-fragmentation of the elastic layers, and thinning out of the media - changes similar to those seen in vascular complications of neurofibromatosis elsewhere in the body. Thus, the multiple anastomoses and characteristic appearance of Moyamoya disease seem to reflect a cerebro-vascular-specific reaction to a variety of arterial changes, one of which may be neurofibromatosis.

\section{References}

Götze, P. \& D. Kühne (1976). Sogenanntes Moyamoya-syndrom (hämangiomartiges Gefassnetz an der Schädelbasis) verbunden mit einer Neurofibromatose von Recklinghausen. Nervenarzt 47, 34-39.

Hilal, S. K., G. E. Solomon, A. P. Gold \& S. Carter (1971a). Primary cerebral occlusive disease in children. Part 1 . Acute acquired hemiplegia. Radiology 99, 71-86.

Hilal, S. K., G. E. Solomon, A. P. Gold \& S. Carter (1971b). Primary cerebral occlusive disease in children. Part II. Neurocutaneous syndromes. Radiology 99, 87-93.

Holt, J. F. (1978). Neurofibromatosis in children. Amer. J. Roentgenol. 130, 615-639.

Lamas, E., R. Diez Lobato, A. Cabello \& J. M. Abad (1978). Multiple intracranial arterial occlusions (Moyamoya disease) in patients with neurofibromatosis. Acta neurochir. 45, 133-145.

Levisohn, P. M., M. A. Mikhael \& S. M. Rothman (1978). Cerebrovascular changes in neurofibromatosis. Develop. Med. Child. Neurol. 20, 789-793. 
Rosenquist, G. C., L. J. Krovetz, J. A. Haller Jr., A. L. Simon \& G. A. Bannayan (1970). Acquired right ventricular outflow obstruction in a child with neurofibromatosis. Amer. Heart I. 79, 103-108.

Rowen, M., T. J. Dorsey, S. M. Kegel \& W. E. Ostermiller Jr. (1975). Thoracic coarctation associated with neurofibromatosis. Amer. $J$. Dis. Child. 129, 113-115.

Seeler, R. A., J. E. Royal, L. Powe \& H. R. Goldbarg (1978). Moyamoya in children with sickle cell anemia and cerebrovascular occlusion. J. Pediat. 93, 808-810.

Søgaard, I. \& J. Jørgensen (1975). Familial occurrence of bilateral intracranial occlusion disease of the internal carotid arteries (MoyaMoya). Acta neurochir. 31, 245-252.
Suzuki, J. \& A. Takaku (1969). Cerebrovascular "Moya-Moya" disease: Disease showing abnormal net-like vessels in base of brain. Arch. Neurol. (Chic.) 20, 288-299.

Tomsick, T. A., R. R. Lukin, A. A. Chambers \& C. Benton (1976). Neurofibromatosis and intracranial arterial occlusive disease. Neuroradiology 11, 229-234.

Address:

Robert P. Erickson, M.D.

Department of Human Genetics

University of Michigan School of Medicine

Ann Arbor

Michigan 48109

U.S.A. 